\title{
Real Time Tracing and Alerting System for Vehicles and Children to Ensure Safety and Security Using Labview
}

\author{
Dr. R. Deepalakshmi ${ }^{\text {a,1 }}$,Dr.R. Vijayalakshmi a, S. Lavanya ${ }^{\text {a }}$, T.K. Rakshitha Rasmi ${ }^{\text {a }}$, \\ S.B. Sathiya ${ }^{\text {a }}$ \\ ${ }^{a}$ Department of Computer Science and Engineering, Velammal College of Engineering and Technology, \\ Madurai, Tamil Nadu, India
}

\begin{abstract}
The Absolute time monitoring, detecting and Alerting System for vehicles and children is required to trace and transmit the collected information at regular intervals to ensure safety and security of children. The illustration of the Realtime detecting and warning System consists of two units: Tracing Unit that traces the location information, transfers to the monitoring area, records the data in the database and takes the help of these data to locate the exact point of area of the vehicle with Google/other maps. The second unit is Alerting Unit that tracks the students using active Radio Frequency Identification Devices (RFID)which will be placed on student ID card. radio- wave trans-receiver transmits a common radio wave which is received by the RFID in the ID card. This radio-wave is modified by the RFID's coil and resent to the receive RFID tags are also used for attendance which is updated directly to the database and displays the other studentinformation.
\end{abstract}

Keywords: Tracking, Laboratory Virtual Instrument Engineering (LabVIEW), Position, Radio Frequency Identification (RFID), Alerting, RFID Tags, RFID reader.

\section{Introduction}

Safety and security are the most important aspects for students and the management to prevent students from abduct, mislaid etc., In the existing passive tracking system, a sensor device is placed in the vehicle that records position, speed etc., and also when vehicle backs to the specified position the device is deleted and data transferred to the system. It also includes automatic downloading option which transfers information through radio link but were not absolute time. Passive system does not help to be more helpful to trace vehicles to prevent accidents. Absolute time tracing system was needed to send the stored information of the vehicle at regular intervals or at least transmit the information when required by the monitoring space. In this system, a device is fixed in the vehicle which will be interlinked with LabView to track the vehicle in real-time, locate it on the Google/other map and the alarming system included with alerting the client about the vehicle location only. This does not concentrate on the passengers in the vehicle. This led to the making of active modules. Hence this automatic vehicle tracing system will help you to track the particular target vehicle as well as the students in real time and also alerts the parents and school management at regular intervals of time by using the

\footnotetext{
${ }^{1}$ Deepalakshmi, R, Professor, Velammal College of Engineering and Technology, India Email: rdl@vcet.ac.in.
} 
software called Lab View for vehicle tracking and RFID for student tracking. The active system is developed for the real time tracking of particular target vehicle at regular interval of time. By using LabView, it captures the image of the vehicle and starts tracking it. The vehicle information is always stored in the database such as time, speed, location which is connected with Google Maps. The students who are travelling in the vehicle are also tracked using RFID. Each student contains a tag that has a unique code. RFID system uses an electromagnetic field to transmit data from RFID to tracker. RFID, indeed provides accurate and real time tracking data for fixed and mobile assets. The vehicle and student information are stored in the database. Alerting system alert the parents and the management by getting information from the database linked whenever required.

\section{System Requirements}

- Browser: Firefox, Edge, Chrome, etc.,

- Operating System: Windows 10 with core i5 processor

- IDLE: LabVIEW version 18.0(32-bit)

\section{Architecture of the project}

The domain of project consists of two modules: The first module is Vehicle tracking, this module tracks the target vehicle in real time by using the software LabView and stores the location information, vehicle video and stores the collected information in database which is interlinked with the software. Vehicle tracing is the process of pointing a moving material in using a lens/camera. The algorithm senses the frames and outputs the position of material within the frame. School Vehicle Tracking System uses LABVIEW software that is used to track the vehicle.

This is the Algorithm of Vehicle tracking using LabVIEW simulation tool:

Step 1: Capture and save the image of the vehicle using the LabVIEW simulation tool

Step 2: Check if the captured vehicle is same as the target vehicle.

a) If YES, track the vehicle until the destination is reached.

b) If NO, capture and check for target vehicle until found.

Step 3: Once the target vehicle is found and tracked, check if the location information is available.

a) If YES, store it in the databaseserver.

b) If $\mathrm{NO}$, again continue with step $2 \mathrm{~b}$.

Step 4: Make sure the location information stored serves the needful and use them track the vehicle in Google maps.

The role of the tracing algorithm is to analyze the frames to calculate the moving measurements. These measurements characterize the position of the target vehicle. They help to identify other factors such as speed, number of route changes, total time moving and also information about size of the target vehicle. 


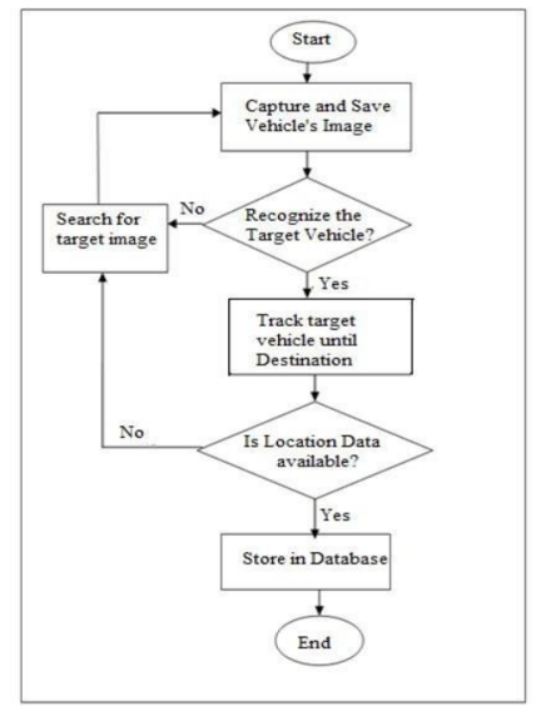

Figure 1. Vehicle tracking system flow diagram

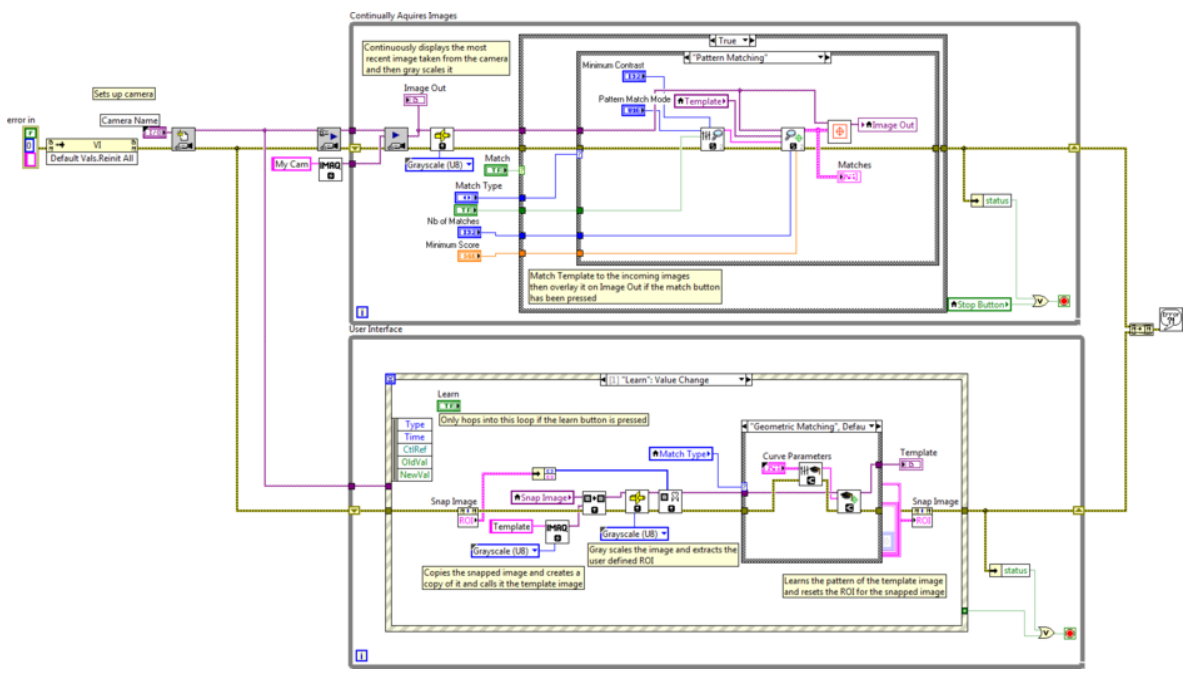

Figure 2. Block Diagram of Vehicle Tracking

The second module is student tracking system. It recognizes the student who enters the vehicle at the source location by reading the tag holds by the student using RFID (Radio Frequency Identification), and monitors the presence and absence of students and stores the information in the database. By integrating this system with DBMS (Database Management System), a database can be maintained to monitor the speed and the location at which the 19 vehicles had travelled. The alerting system can also be provided in case of any speed violation by the traveler. The speed limit can be set by the owner of the vehicle and they can receivenotifications through mail in case the vehicle travels above the speed limit specified by the user. 
The following is the Algorithm for student tracking using RFID Tag:

Step 1: When the student enters the bus, the RFID tag details are recognized by the sensor and the data is sent to the RFIDreader.

Step 2: This reader decrypts the message and sends it the Attendance application.

Step 3: The Attendance application compares the information with the database.

Step 4: Checks if the data matches with the Database

a) If YES, Attendance is marked, saved and acknowledged.

b) If NO, Absence is marked and notification is sent to the parents and alerts them.

Step 5: Parents are acknowledged on students reaching the destination.

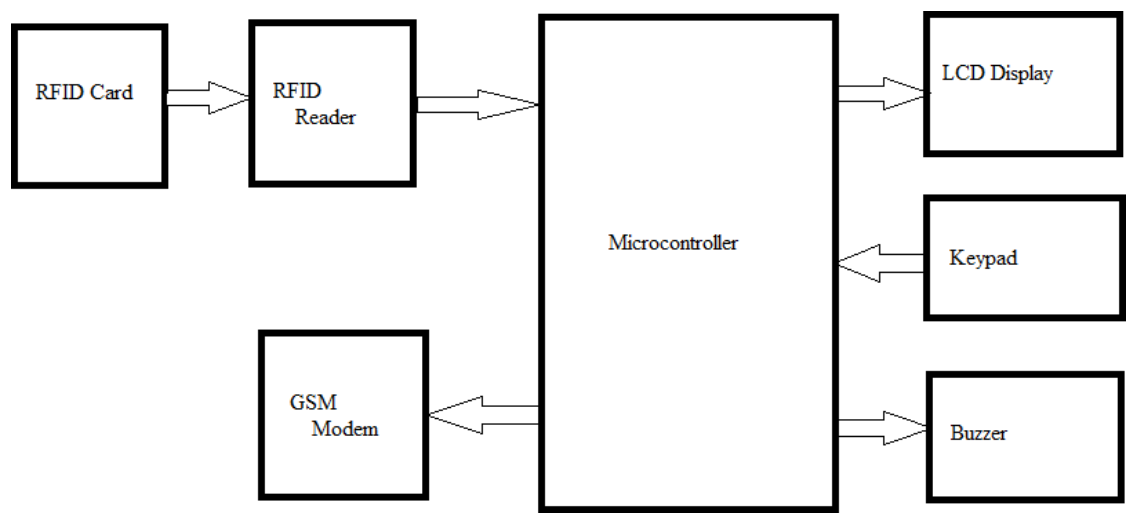

Figure 3. Student Tracking and Attendance System

\section{Module}

1. Vehicle Tracking using LabVIEW.

2. Student Tracking using RFID.

3. Alerting System

\subsection{Vehicle Tracking using LabVIEW}

This module tracks the vehicle using LabVIEW. LabVIEW is just a simulation tool. LabVIEW (Laboratory Virtual Instrument Engineering Workbench) is a modelcreating platform. It is created by National Instruments (NI). LabVIEW is used for automated manufacturing, test, validation, take charge and monitoring of a system. For real time tracking of vehicle, LabVIEW captures the image of the target vehicle by using the in- built camera and then highlights the captured image using rectangular red box. Once, when the LabVIEW diagram and the vehicle image gets simulated it starts tracking the vehicle until it reaches the destination. While tracking the vehicle, information like vehicles location and time information gets stored in the database. 

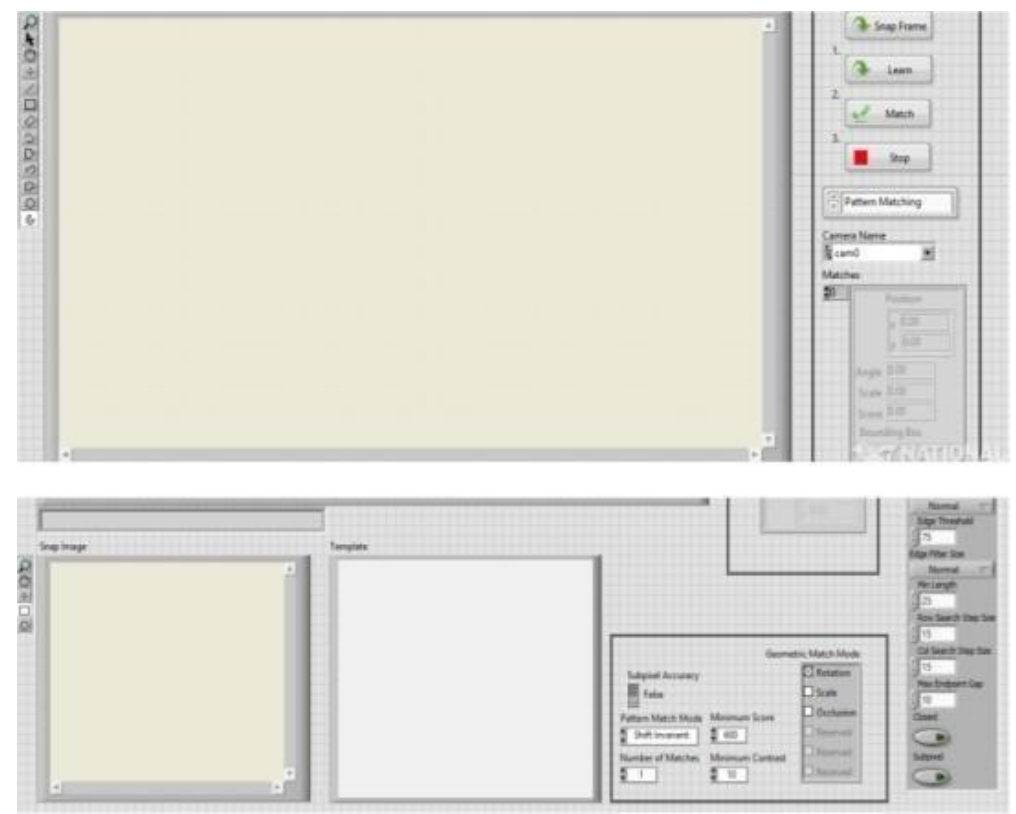

Figure 4. LabVIEW Implementation

\subsection{Student tracking using RFID}

Parents need ways to monitor the safety of their children during their journey to/from school. In this module, Student get tracked by using the technology RFID. RFID is used for data collecting that includes automatic identification of materials using tags, an antenna, an RFID reader and a transceiver.

Step 1: Wearable RFID tags are given to all students.

Step2: RFID reader are installed in the vehicle. When the student enters the bus, it automatically scans the reader.

Step3: RFID identifies the tag number and stores the student information such as students source location, absence of students and the entering time the database.

\subsection{Alerting system}

Collected information from the first module is stored in the vehicle database, and the information about the students are stored in relevant ID. In the module, a solution is provided using Radio Frequency Identification (RFID) technology in order to track students journey to/from school thus notifies the parents about the status of children. This reads the children's RFID tag and automatically sends different generated messages to their parents. The proposed solution has a set of capabilities, such as notifying the parents that the children has arrived to the school safely, and if any children are absent without an authorized permission then an immediate message is sent to their parents. 


\section{Conclusion}

The system mainly aims and concentrates on making a system to trace the location of vehicle and the passenger in absolute time, point it on the Google/other maps and alarming module added to heed user of the module if diversion/interruption in route takesplace as well as when students are missing/absence of students. The system could yet be used to trace vehicles and alert passengers from misfortune, also provide emergency/fast services in case of any such situations. This system can be expanded to identify vehicles which looks similar to each other by using their number plate, the student tracing can be done more precisely to avoid misusing of tags by students and also calculating the Body Mass Index of an individual for identifying the particular student.

\section{References}

[1] Dao, D., Rizos, Ch., Wang, J., (2002).Location-based services: technical and business issues, GPS Solutions Vol.6.

[2] David Moore,(June 5,2003). A real-world system for human motion detection and tracking, California Institue of Technology.

[3] Prawat Chaiprapa, Supaporn Kiattisin and Adisorn Leelasantitham ,(2003). A Real-Time GPS Vehicle Tracking System Displayed on a Google- Map-Based.

[4] Guochang Xu,(Springer 2007).GPS Theory,Algorithms and Applications.

[5] F. M. Franczyk, and J. D. Vanstone, (22 April 2008). Vehicle warning system, Patent number: 7362239.

[6] Thuong Le-Tien, Vu Phung, (July,2009).Routing and Tracking System for Mobile Vehicles in Large Area,Vietnam.

[7] ThuongLe-Tien, Vu Phung, (IEEE,2010).Routing and Tracking System for Mobile Vehicles in Large Area,Vietnam.

[8] Reddy, S. S., (2011).Trip Tracker Application on Android, San Diego State University, USA.

[9] Dinkar, A. Sh., Shaikh, S. A., (2011). Design and Implementation of Vehicle Tracking System Using GPS, Journal of information Engineering and Applications.

[10] Harshadbhai, P. K., (2013).Design of GPS and GSM Based Vehicle Location and Tracking System, International Journal of Science and Research,Vol. 2,No. 2,pp. 165-168.

[11] Lee, S., Tewolde, G., Kwon, J.,(IEEE 2014),.Design and implementation of vehicle tracking system using GPS/GSM/GPRS technology and smart phone application. 